\title{
Wnt7A is a putative prognostic and chemosensitivity marker in human malignant pleural mesothelioma
}

\author{
TOMOMI HIRATA ${ }^{1,2^{*}}$, QINGFENG ZHENG ${ }^{1,3^{*}}$, ZHAO CHEN $^{1,4}$, HIROYASU KINOSHITA $^{1,2}$, \\ JUNICHI OKAMOTO ${ }^{1,2}$, JOHANNES KRATZ ${ }^{1}$, HUI LI ${ }^{1}$, NATALIE LUI ${ }^{1}$, HANH DO ${ }^{1}$, TIFFANY CHENG ${ }^{1}$, \\ HSIN-HUI KATTY TSENG ${ }^{1}$, KIYOSHI KOIZUMI ${ }^{2}$, KAZUO SHIMIZU ${ }^{2}$, HAI-MENG ZHOU ${ }^{4}$, \\ DAVID JABLONS ${ }^{1,5}$ and BIAO HE $\mathrm{HE}^{1,5}$ \\ ${ }^{1}$ Thoracic Oncology Program, Department of Surgery, University of California, San Francisco, CA 94115, USA; \\ ${ }^{2}$ Department of Surgery, Division of Thoracic Surgery, Nippon Medical School, Tokyo 113-8602, Japan; \\ ${ }^{3}$ Key Laboratory of Carcinogenesis and Translational Research (Ministry of Education), Thoracic Surgery II, \\ Peking University Cancer Hospital and Institute, Beijing 100142; ${ }^{4}$ School of Life Sciences, \\ Tsinghua University, Beijing 100084, P.R. China; ${ }^{5}$ Helen Diller Family Comprehensive \\ Cancer Center, University of California, San Francisco, CA 94143, USA
}

Received October 29, 2014; Accepted December 8, 2014

DOI: $10.3892 /$ or.2015.3771

\begin{abstract}
Malignant pleural mesothelioma (MPM) is a highly aggressive tumor that has a poor prognosis, limited treatment options, and a worldwide incidence that is expected to increase in the next decade. We evaluated Wnt7A expression in 50 surgically resected tumor specimens using quantitative PCR. The expression values, were assessed by clinicopathological factors and K-M and Cox's regression with OS. The mean level of Wnt7A expression had a significant correlation with International Mesothelioma Interest Group (IMIG) stage $(\mathrm{P}<0.034)$, gender, smoking history and ethnicity, respectively $(\mathrm{P}=0.020, \mathrm{P}=0.014, \mathrm{P}=0.039)$. In the univariate analysis, low Wnt7A expression was a significant negative factor for overall survival $(\mathrm{P}=0.043, \mathrm{HR}=2.30)$. However, multivariate Cox's regression revealed no significant factors for overall survival (low Wnt7A: $\mathrm{P}=0.051, \mathrm{HR}=2.283$; non-epithelioid subtype: $\mathrm{P}=0.050, \mathrm{HR}=2.898)$. In patients with epithelioid tumors, those with low Wnt7A expression had significantly worse prognosis $(\mathrm{P}=0.019, \mathrm{HR}=2.98)$. In patients with epithelioid tumors, females had significantly better prognosis than males $(\mathrm{P}=0.035)$. In patients who did not have neoadjuvant chemotherapy, prognosis was significantly more favorable for patients with high $W n t 7 A$ expression than for those with low $W n t 7 A$ expression $(\mathrm{P}=0.031)$. Among the patients with low Wnt7A-expressing tumors, those who received neoadju-
\end{abstract}

Correspondence to: Dr Biao He, Thoracic Oncology Program, Department of Surgery, Helen Diller Family Comprehensive Cancer Center, University of California, 2340 Sutter Street, San Francisco, CA 94115, USA

E-mail: biao.he@ucsfmedctr.org

*Contributed equally

Key words: mesothelioma, marker, chemotherapy vant chemotherapy had better prognosis than those who did not $(\mathrm{P}=0.024)$. The results of our study suggest that $W n t 7 A$ expression is a putative prognostic factor and a predictor of chemosensitivity.

\section{Introduction}

Malignant pleural mesothelioma (MPM) is an asbestosinduced, highly aggressive tumor that was once considered rare, but its incidence has steadily increased and new cases are predicted to peak between 2015 and $2025(1,2)$. The disease is almost always fatal; median survival is 9 months for patients treated with supportive care, 12.1 months for those who receive the best available chemotherapy (3), and 11.7-13 months for those who have maximal cytoreductive surgery, as well as chemotherapy and/or radiation $(4,5)$. The treatment with chemotherapy has shown slightly improved survival, and multi-modal trials are ongoing (1). A previous study has revealed that pemetrexed plus cisplatin neoadjuvant chemotherapy followed by extrapleural pneumonectomy (EPP) and hemithoracic radiation improved the 2-year overall survival rate (6).

Known positive prognostic factors for MPM include good performance status, young age, female, early stage and epithelioid subtype (7-10). Several markers have been proposed either as positive (e.g. Syndecan 1 expression) $(11,12)$ or negative (e.g. Cox-2 expression) (13), and new microarray technology currently reveals batteries of genes that may have prognostic value $(14,15)$. Although the two principal tumor suppressor genes, $R b$ (16-18) and $p 53$ (19), are not commonly absent in MPM, other molecules that are important in the $R b$ and $p 53$ pathways are present in MPM (1), particularly p16 and pl4 $(20,21)$. However, whether tumor suppressor expression is correlated with MPM prognosis is not known. Members of the Wnt signaling family are highly conserved and secrete glycoproteins vital to mammalian development and many aspects of adult tissue homeostasis. The 
members of this family regulate important cellular processes, such as proliferation, differentiation and cell fate specification (22). Furthermore, the Wnt gene family encodes transcription factors that regulate morphogenesis and cell differentiation during embryogenesis by activating or repressing the expression of target genes (23). Wnt signals are transduced through 7 transmembrane-type Wnt receptors encoded by Frizzled (Fz) genes to the $\beta$-catenin-TCF pathway, the JNK pathway or the $\mathrm{Ca}^{2+}$-releasing pathway. E-cadherin induction by $\mathrm{Wnt} / \beta$-catenin signaling is an evolutionarily conserved pathway operative in lung cancer cells, and loss of Wnt7A expression may be important in lung cancer development or progression due to its effects on E-cadherin (24). Therefore, Wnt7A appears to play important roles in embryonic development and tumorigenesis (25-27). Wnt signaling molecules are potent targets for cancer diagnosis (susceptibility, metastasis and prognosis), cancer prevention and treatment, and for regenerative medicine or tissue engineering (28).

We therefore analyzed Wnt7A expression by using quantitative RT-PCR in surgically resected MPM specimens and/or adjacent normal tissues. We also examined the correlations between $W n t 7 A$ expression and various clinicopathological factors, prognosis and neoadjuvant chemotherapy.

\section{Materials and methods}

Patients. Between 1999 and 2005, 50 fresh samples of MPM and/or the adjacent normal tissues were collected from consecutive patients undergoing surgical resection in a study approved by the Committee on Human Research at the University of California, San Francisco (UCSF). Of these 50 patients, 24 had received neoadjuvant chemotherapy before surgery. The pathologic classification of each sample was confirmed by a review of sections stained with hematoxylin and eosin. Clinical information was also reviewed: age, gender, ethnicity, smoking status, Eastern Cooperative Oncology Group performance status (ECOG PS), histological subtype, International Mesothelioma Interest Group (IMIG) stage, surgical procedure, chemotherapy, radiation, recurrence status, vital status, progression status, and overall survival, which was calculated from the date of surgery.

Tissues and RNA extraction. Tissue samples were promptly snap-frozen in liquid nitrogen and stored at $-170^{\circ} \mathrm{C}$ before use Total RNA was extracted using TRIzol LS (Invitrogen) and purified using the RNeasy Mini kit (Qiagen).

Quantitative real-time reverse transcription-PCR. cDNA synthesis and Taqman PCR were performed as previously described (29). Hybridization probes and primers (Wnt7A, Hs01114990 m1; GAPDH, Hs00244574 m1) were purchased from Applied Biosystems (ABI). Wnt7A expression was assayed in triplicate using an ABI 7300 real-time PCR system. Samples were normalized to the housekeeping gene $G A P D H$, and expression levels were calculated using the $2^{-\Delta \Delta \mathrm{Ct}}$ method compared to total RNA of the mixed adjacent normal pleural tissues derived from 11 patients.

Statistical analysis. Patients were divided into those with high Wnt7A expression and those with low Wnt7A expression. These groups were compared with respect to the clinicopathological factors by using t-tests, Chi-square tests or Kaplan-Meier survival curves and the Log-rank test. A correlation between Wnt7A expression and IMIG staging system [stage, primary site $(\mathrm{T})$ or lymph node metastasis $(\mathrm{N})$ ] was analyzed by one-way ANOVA. Kaplan-Meier survival curves for overall survival were compared for various clinicopathologic factors. Prognostic variables that were significant on univariate analysis were entered into a Cox's proportional hazards model to determine the hazard ratio (HR). The cutoff values were associated with the lowest $\mathrm{P}$ obtained when comparing the two Wnt7A expression groups (the optimal $\mathrm{P}$ approach). Two-sided P-values $<0.05$ were considered significant. All analyses were conducted using the IBM SPSS statistics software package, ver. 18.0.

\section{Results}

Patient characteristics. Table I summarizes the characteristics of the 50 patients and prognosis after surgery according to Wnt7A expression (high Wnt7A, $\mathrm{n}=30$; low Wnt7A, $\mathrm{n}=20$ ). Performance status data were missing for 26 patients. The Wnt7A expression groups differed significantly in regards to gender, smoking status, performance status and vital status.

Univariate analyses. In the univariate analysis for overall survival, gender, performance status, subtype and Wnt7A expression were all statistically significant (Table II). Worse overall survival correlated with low Wnt7A expression when expression was assessed as a categorical variable $[\geq 4.315$ $\left(\log _{10}(\right.$ Wnt7A expression $)=0.635, \mathrm{P}=0.043 ; \mathrm{HR}=2.30(95 \% \mathrm{CI}$, 1.01-5.25)].

Multivariate analysis. Multivariate analysis using a Cox's regression hazard model showed no significant prognostic factors for overall survival, but did show that low Wnt7A expression $(\mathrm{P}=0.051, \mathrm{HR}=2.283)$ and histological subtype (non-epithelioid, $\mathrm{P}=0.05, \mathrm{HR}=2.898$ ) were likely to be negative prognostic factors for overall survival (Table III).

Wnt7A expression and its relationship with clinicopathological characteristics and overall survival. Wnt7A expression differed significantly between the 11 paired normal and tumor tissues ( $\mathrm{P}=0.005$, Fig. 5), and by tumor stage (Fig. 1A), gender (Fig. 1B), smoking status (Fig. 1C) and ethnicity (Fig. 1D). The logarithm of Wnt7A expression values in the tumors ranged from -0.58 to +0.89 (mean $\pm \mathrm{SD}, 0.98 \pm 0.14$ ). The frequency distribution graph showed two peaks (Fig. 6), which allowed two subgroups, high-risk (low Wnt7A) and low risk (high $W n t 7 A$ ) to be defined. A cutoff point for distribution of Wnt7A expression into 2 groups was optimized at 4.67 in the logarithm of $W n t 7 A$ expression to acquire a minimum P-value in the difference of overall survival between the 2 groups.

Overall survival was significantly more favorable for patients with high Wnt7A expression than for those with low expression (26.7 \pm 11.1 months vs. 11.8 $\pm 4.9 ; \mathrm{P}=0.043$, Fig. 2A). To confirm a correlation between $W n t 7 A$ expression and survival, we divided the distribution of Wnt7A expression into thirds (low Wnt7A, intermediate Wnt7A, high Wnt7A) using the $33 \mathrm{rd}$ and 66 th percentiles. We found that Kaplan-Meier 
Table I. Clinicopathological characteristics of the patients with malignant pleural mesothelioma, according to Wnt7A expression.

\begin{tabular}{|c|c|c|c|c|}
\hline & $\begin{array}{l}\text { Total } \\
(\mathrm{n}=50)\end{array}$ & $\begin{array}{c}\text { High } \\
W n t 7 A \\
(\mathrm{n}=30)\end{array}$ & $\begin{array}{c}\text { Low } \\
W n t 7 A \\
(\mathrm{n}=20)\end{array}$ & P-value \\
\hline \multicolumn{5}{|l|}{ Age (years) } \\
\hline Mean \pm SD & $66 \pm 9$ & $67 \pm 10$ & $67 \pm 9$ & 0.942 \\
\hline Range & $(45-84)$ & $(59-84)$ & $(54-84)$ & \\
\hline \multicolumn{5}{|l|}{ Gender } \\
\hline Male/female & $41 / 9$ & $21 / 9$ & $20 / 0$ & 0.007 \\
\hline \multicolumn{5}{|l|}{ Ethnicity } \\
\hline Caucasian/other & $38 / 12$ & $25 / 5$ & $13 / 7$ & 0.182 \\
\hline \multicolumn{5}{|l|}{ Smoking } \\
\hline Yes/no & $16 / 24$ & $14 / 11$ & $2 / 13$ & 0.024 \\
\hline \multicolumn{5}{|l|}{ ECOG PS } \\
\hline $0 / 1 / 2 / \mathrm{ND}$ & $10 / 13 / 1 / 26$ & $6 / 4 / 0 / 20$ & $4 / 9 / 1 / 6$ & 0.026 \\
\hline \multicolumn{5}{|l|}{ Subtype } \\
\hline Epithelioid/other & $42 / 8$ & $25 / 5$ & $17 / 3$ & 0.875 \\
\hline \multicolumn{5}{|l|}{ IMIG stage } \\
\hline I/II/III/IV & $1 / 18 / 24 / 5$ & $1 / 13 / 13 / 2$ & $0 / 5 / 11 / 3$ & 0.363 \\
\hline \multicolumn{5}{|l|}{ Primary tumor $(\mathrm{T})$} \\
\hline $\mathrm{T} 1 / \mathrm{T} 2 / \mathrm{T} 3 / \mathrm{T} 4$ & $1 / 20 / 22 / 5$ & $1 / 16 / 11 / 3$ & $0 / 4 / 11 / 2$ & 0.550 \\
\hline \multicolumn{5}{|l|}{$\begin{array}{l}\text { Regional lymph } \\
\text { node }(\mathrm{N})\end{array}$} \\
\hline $\mathrm{N} 0 / \mathrm{N} 1 / \mathrm{N} 2$ & $37 / 5 / 6$ & $24 / 1 / 4$ & $13 / 4 / 2$ & 0.293 \\
\hline \multicolumn{5}{|l|}{ Surgical procedure } \\
\hline EPP/PLE/CWR/BIO & $11 / 35 / 2 / 2$ & $8 / 19 / 2 / 1$ & $3 / 16 / 0 / 1$ & 0.451 \\
\hline \multicolumn{5}{|l|}{ Chemotherapy } \\
\hline Yes/no & $30 / 20$ & $18 / 12$ & $12 / 8$ & 1.000 \\
\hline Neo/PSC/both & $24 / 8 / 2$ & $15 / 4 / 1$ & $11 / 4 / 1$ & 0.529 \\
\hline Neo, yes/no & $24 / 26$ & $15 / 15$ & $9 / 11$ & 0.447 \\
\hline PSC, yes/no & $8 / 42$ & $4 / 26$ & $4 / 16$ & 0.351 \\
\hline \multicolumn{5}{|l|}{ Radiation } \\
\hline Yes/no & $20 / 30$ & $12 / 18$ & $8 / 12$ & 0.162 \\
\hline \multicolumn{5}{|l|}{ Progression } \\
\hline Yes/no & $19 / 31$ & $17 / 13$ & $12 / 8$ & 0.525 \\
\hline \multicolumn{5}{|l|}{ Vital status } \\
\hline Alive/deceased & $25 / 25$ & $19 / 11$ & $6 / 14$ & 0.042 \\
\hline \multicolumn{5}{|l|}{ Overall survival (OS) } \\
\hline Median OS time & $14.5 \pm 4.5$ & $26.7 \pm 11.2$ & $11.8 \pm 1.0$ & 0.043 \\
\hline
\end{tabular}

ECOG PS, Eastern Cooperative Oncology Group performance status; ND, not defined; IMIG, International Mesothelioma Interest Group; EPP, extrapleural pneumonectomy; PLE, pleurectomy; CWR, chest wall resection; BIO, biopsy; Neo, neoadjuvant chemotherapy; PSC, postsurgical chemotherapy.

curves plotted for epithelioid tumors showed better prognosis $(\mathrm{P}=0.023$, Fig. 6). Previously Fennel et al estimated in a similar way (30).
Table II. Univariate analysis of overall survival in patients with malignant pleural mesothelioma.

\begin{tabular}{|c|c|c|c|}
\hline & \multicolumn{3}{|c|}{ Overall survival } \\
\hline & P-value & Hazard ratio & $95 \% \mathrm{CI}$ \\
\hline \multicolumn{4}{|l|}{ Age (average, $66 \pm 9$ ) } \\
\hline$\leq 66 />66$ years & 0.347 & $1 / 1.47$ & $(0.66-3.29)$ \\
\hline \multicolumn{4}{|l|}{ Gender } \\
\hline Male/female & 0.096 & $1 / 0.46$ & $(0.17-1.26)$ \\
\hline \multicolumn{4}{|l|}{ Ethnicity } \\
\hline Caucasian/other & 0.242 & $1 / 1.63$ & $(0.71-3.71)$ \\
\hline \multicolumn{4}{|l|}{ Smoking } \\
\hline No/yes & 0.163 & $1 / 1.99$ & $(0.74-5.40)$ \\
\hline \multicolumn{4}{|l|}{ ECOG PS } \\
\hline $0 / 1+2$ & 0.01 & $1 / 3.89$ & $(1.29-11.8)$ \\
\hline \multicolumn{4}{|l|}{ Surgical procedure } \\
\hline EPP/PLE/CWR/BIO & 0.056 & $0 / 2.44 / 0 / 1$ & $\begin{array}{c}\text { PLE } \\
(0.33-18.32)\end{array}$ \\
\hline \multicolumn{4}{|l|}{ IMIG staging system } \\
\hline $1+2 / 3+4$ & 0.181 & $1 / 1.754$ & $(0.76-4.04)$ \\
\hline \multicolumn{4}{|l|}{ Primary tumor $(\mathrm{T})$} \\
\hline $\mathrm{T} 1+\mathrm{T} 2 / \mathrm{T} 3+\mathrm{T} 4$ & 0.241 & $1 / 1.63$ & $(0.72-3.68)$ \\
\hline \multicolumn{4}{|l|}{ Regional lymph node } \\
\hline $\mathrm{N}^{-} / \mathrm{N}^{+}$ & 0.816 & $1 / 1.13$ & $(0.382-3.390)$ \\
\hline \multicolumn{4}{|l|}{ Subtype } \\
\hline Epithelioid/other & 0.038 & $1 / 2.91$ & $(1.01-3.34)$ \\
\hline \multicolumn{4}{|l|}{$\begin{array}{l}\text { Neoadjuvant } \\
\text { chemotherapy }\end{array}$} \\
\hline Yes/no & 0.423 & $1 / 1.14$ & $(0.63-3.03)$ \\
\hline \multicolumn{4}{|l|}{$\begin{array}{l}\text { Adjuvant } \\
\text { chemotherapy }\end{array}$} \\
\hline Yes/no & 0.143 & $1 / 0.47$ & $(0.17-1.32)$ \\
\hline \multicolumn{4}{|l|}{ Radiation therapy } \\
\hline Yes/no & 0.395 & $1 / 0.71$ & $(0.32-1.58)$ \\
\hline \multicolumn{4}{|l|}{ Wnt7A expression } \\
\hline High/low & 0.043 & $1 / 2.30$ & $(1.01-5.25)$ \\
\hline $\begin{array}{l}\mathrm{CI} \text {, confidence interval; EC } \\
\text { performance status; EPP, } \\
\text { tomy; CWR, chest wall } \\
\text { Mesothelioma Interest Gr } \\
\text { lymph node metastasis-po }\end{array}$ & $\begin{array}{l}\text { G PS, Ea } \\
\text { xtrapleura } \\
\text { esection; } \\
\text { up; } \mathrm{N}^{-}, \mathrm{lyr} \\
\text { tive. }\end{array}$ & $\begin{array}{l}\text { stern Cooperativ } \\
1 \text { pneumonecton } \\
\text { 3IO, biopsy; IN } \\
\text { nph node metas }\end{array}$ & $\begin{array}{l}\text { Oncology Group } \\
\text { y; PLE, pleurec- } \\
\text { IG, International } \\
\text { sis-negative; } \mathrm{N}^{+} \text {, }\end{array}$ \\
\hline
\end{tabular}

Overall survival was also significantly more favorable for patients with epithelioid tumors than for those with nonepithelioid tumors $(\mathrm{P}=0.038)$. In the 42 patients who had epithelioid tumors, overall survival was significantly worse when the tumors had low Wnt7A expression (Fig. 1C). MST of high $W n t 7 A$ was $25 \pm 5.2$ (95\% CI, 8.8 to 41.2 ) months, whereas MST of low Wnt7A was $10.4 \pm 2.6$ (95\% CI, 5.2 to 15.6) months.

All 9 female patients with MPM had high Wnt7A expression. They also had a more favorable overall survival than 
Table III. Results of the multivariate analysis Cox's regression hazard model for overall and progression-free survival.

\begin{tabular}{|c|c|c|c|c|c|c|c|}
\hline & \multirow[b]{2}{*}{$\beta$} & \multirow[b]{2}{*}{ SE of $\beta$} & \multirow[b]{2}{*}{ Wald } & \multirow[b]{2}{*}{ P-value } & \multirow[b]{2}{*}{ HR } & \multicolumn{2}{|c|}{$95 \% \mathrm{CI}$ for $\mathrm{HR}$} \\
\hline & & & & & & Lower & Upper \\
\hline Non-epithelioid & 1.064 & 0.544 & 3.827 & 0.05 & 2.898 & 0.998 & 8.416 \\
\hline Low Wnt7A & 0.825 & 0.422 & 3.824 & 0.051 & 2.283 & 0.998 & 5.22 \\
\hline
\end{tabular}

95\% CI, 95\% confidence interval; HR, hazard ratio; SE, standard error; Wald, Wald statistic for logistic regression algorithms. ECOG PS was not included in this multivariate analysis.
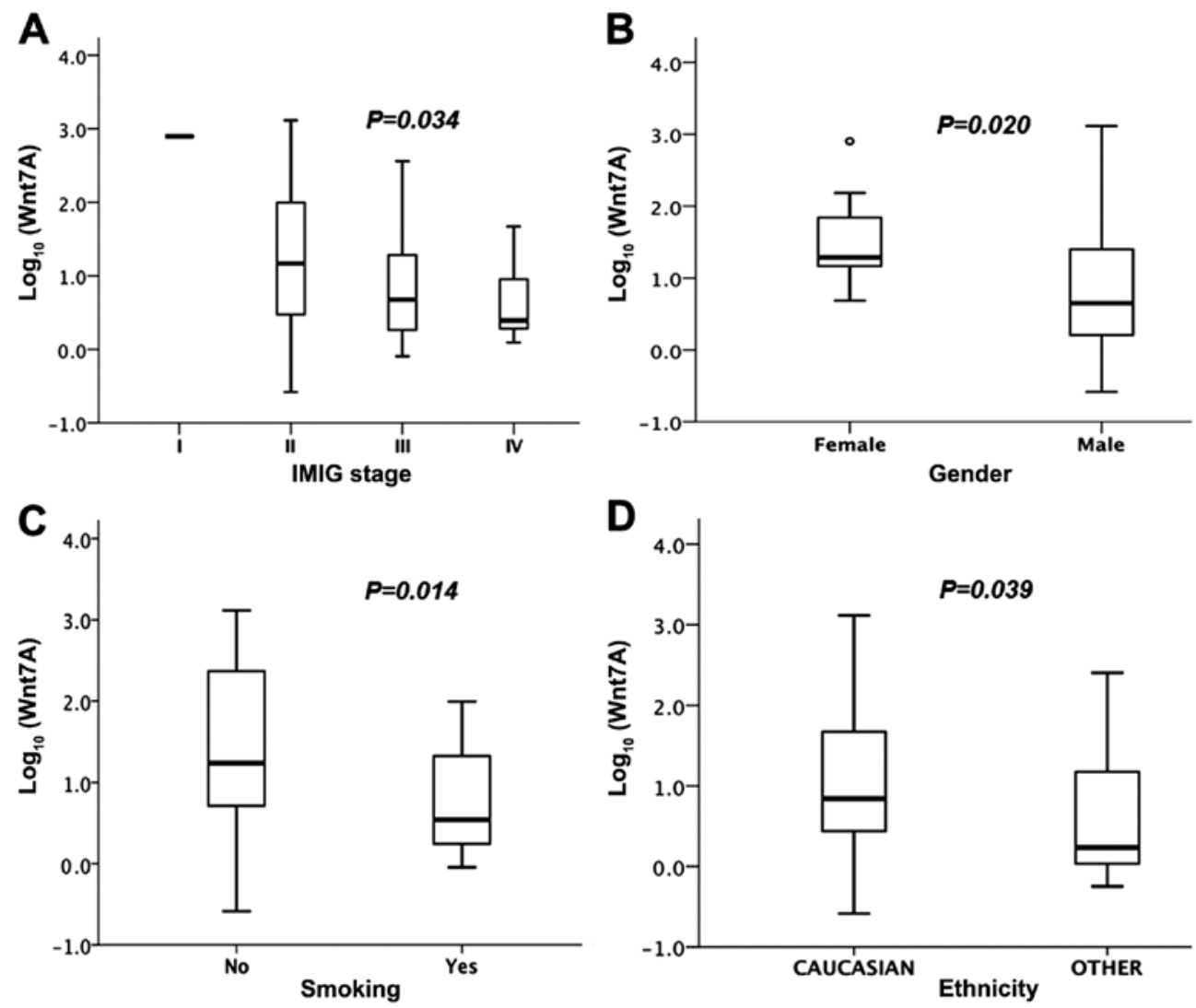

Figure 1. Wnt7A expression logarithm according to clinicopathological factors. (A) IMIG stage (I, II, III, IV), (B) gender, (C) smoking status and (D) ethnicity (Caucasian vs. other ethnicities). P-values were calculated by ANOVA in A and by 2-sided exact test in B-D, respectively.

A

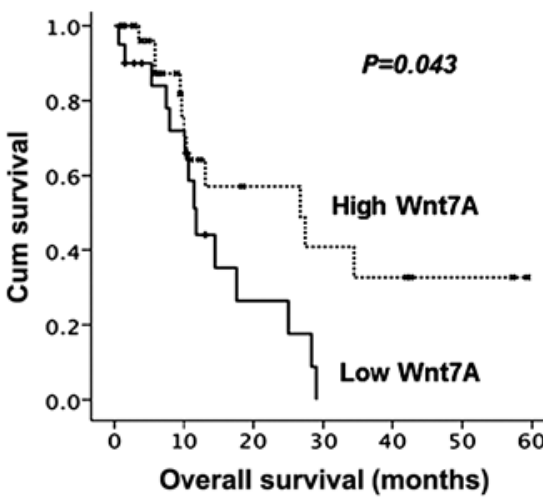

B

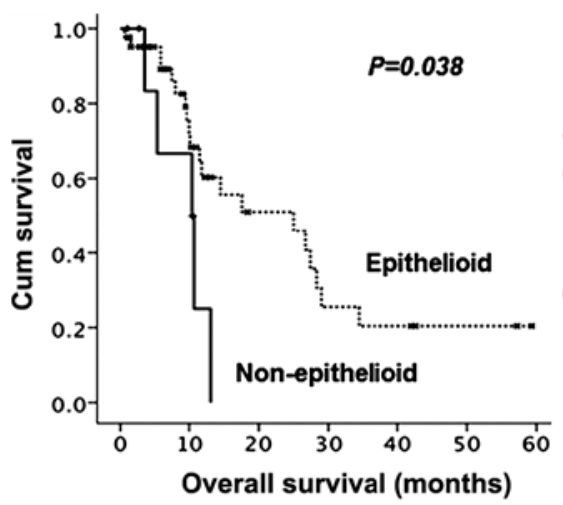

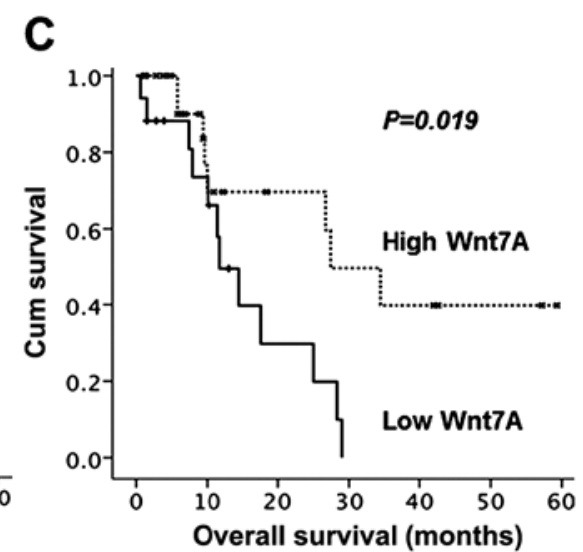

Figure 2. Kaplan-Meier survival curves stratified by Wnt7A expression. (A) Overall survival of all patients according to Wnt7A expression (n=50). (B) Overall survival of all patients according to histological subtype (epithelioid vs. non-epithelioid). (C) Overall survival of patients with epithelioid tumors according to Wnt7A expression. 
A
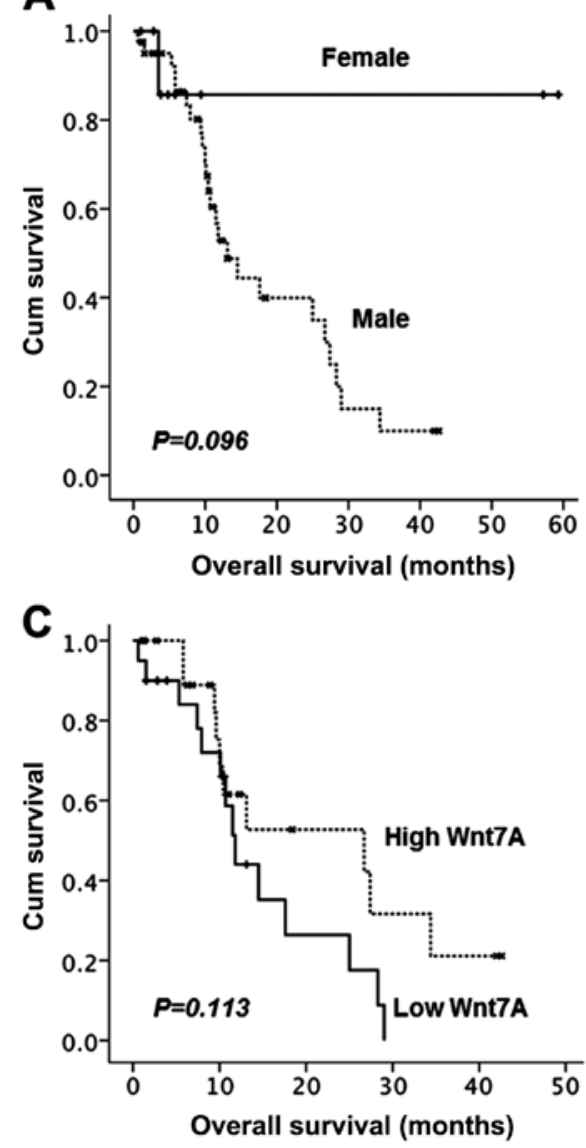

B
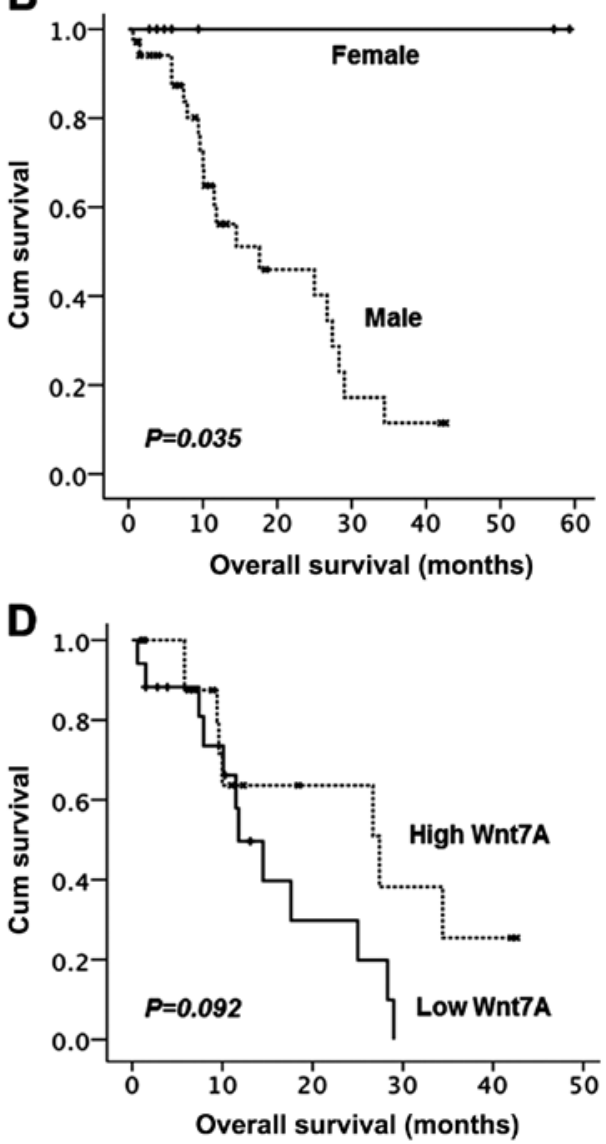

Figure 3. Kaplan-Meier survival curves according to gender and Wnt7A expression. (A) Overall survival of all patients (n=50) according to gender. (B) Overall survival of patients with epithelioid tumors $(\mathrm{n}=42)$ according to gender. $(\mathrm{C})$ Overall survival of male patients $(\mathrm{n}=41)$ according to Wnt7A expression. (D) Overall survival of male patients with epithelioid tumors $(\mathrm{n}=35)$ according to Wnt7A expression.

the male patients $(\mathrm{P}=0.096$, Fig. 3A), including the subgroup with epithelioid tumors ( $\mathrm{P}=0.034$, Fig. 3B). Among the men with epithelioid tumors, those with high Wnt7A expression had better survival than those with low Wnt7A expression $(\mathrm{P}=0.092$, Fig. 3D).

Survival analysis of the 42 patients with epithelioid tumors showed that overall survival for the 20 patients who received neoadjuvant chemotherapy was not significantly better than that for the 22 patients who did not (Fig. 4A). Overall survival was significantly better for patients with high Wnt7A-expressing epithelioid tumors than for those with low Wnt7A-expressing tumors $(27.4 \pm 0$ vs. $10.1 \pm 1.7$ vs. $10.1 \pm 1.7$; $95 \%$ CI, 6.6 to 13.6; $\mathrm{P}=0.019$, Fig. 4B). In the patients with high Wnt7A-expressing epithelioid tumors, overall survival did not differ between those who underwent neoadjuvant chemotherapy and those who did not $(\mathrm{P}=0.902$, Fig. 4C). However, in patients with low Wnt7A-expressing epithelioid tumors, overall survival was significantly better in those who underwent neoadjuvant chemotherapy than in those who did not $(\mathrm{P}=0.024 ; \mathrm{HR}=4.31$, 95\% CI of HR, 1.1 to 16.9 ; Fig. 4E). In the subset of the 20 patients who received neoadjuvant chemotherapy, overall survival did not differ significantly between those with low Wnt7A vs. high Wnt7A tumors $(17.6 \pm 5.25$; 95\% CI, 7.3 to 27.9 months vs. $34.4 \pm 13.3$; $95 \% \mathrm{CI}, 8.2$ to 60.5 months, $\mathrm{P}=0.425$; Fig. 4D). In the subset of 22 patients who did not have neoadjuvant chemotherapy, overall survival was significantly better for patients with high $W n t 7 A$-expressing tumors than for those with low Wnt7A-expressing tumors $(\mathrm{P}=0.031 ; 95 \% \mathrm{HR}=1.03$ to 15.06 ; Fig. $4 \mathrm{~F})$.

\section{Discussion}

The Wnt genes compose a large gene family encoding a group of secreted signaling molecules that have been implicated in oncogenesis and a number of developmental processes. Expression of $W n t 7 A$ is restricted to certain tissues: placenta, kidney, testis, uterus, fetal lung and fetal and adult brain. Why study it in MPM? Our PCR analysis of Wnt7A expression in MPM showed that low Wnt7A expression $(\mathrm{P}=0.051$, $\mathrm{HR}=2.283$ ) and histological subtype (non-epithelioid, $\mathrm{P}=0.05$, $\mathrm{HR}=2.898$ ) were likely to be negative prognostic factors for overall survival. Our survival analyses indicated that Wnt7A expression was correlated with overall survival in the univariate analysis, but not in the multivariate Cox's regression.

In our study, Wnt7A expression was significantly higher in women with MPM than in men (Fig. 1B), and gender was a positive prognostic factor in patients with epithelioid tumors (Fig. 3B). Wnt7A is required for proper differentiation and gland formation during uterine development. Following postnatal growth, Wnt7A expression becomes restricted primarily to the luminal epithelium and is responsible for maintaining expression of other Wnts in the stroma (31-33). The Wnt7A gene 
A

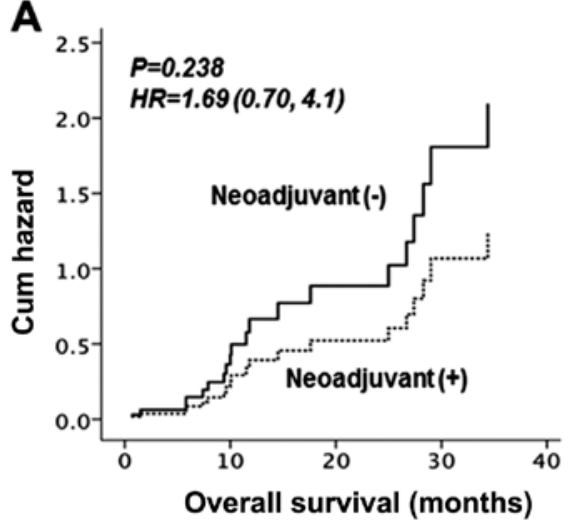

C

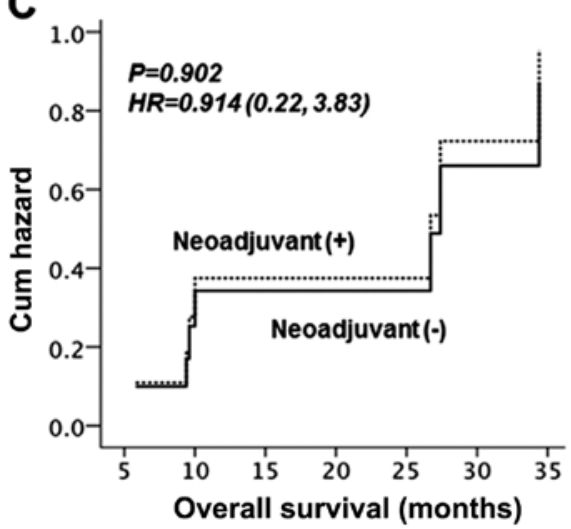

E

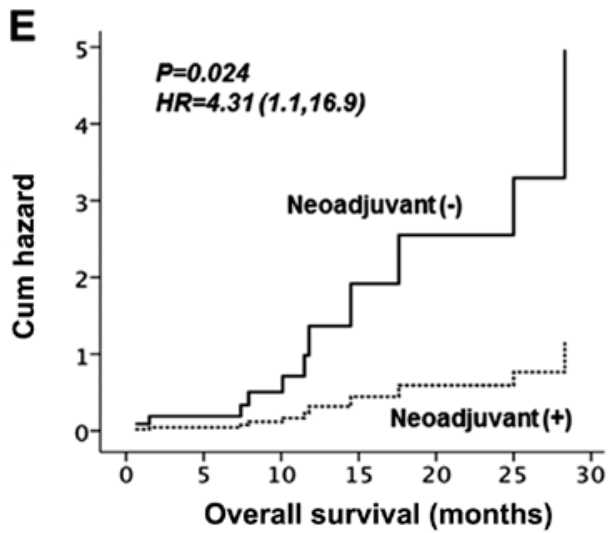

B

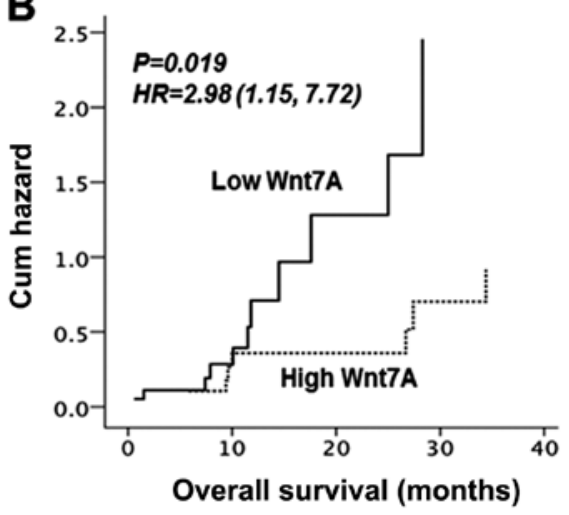

D

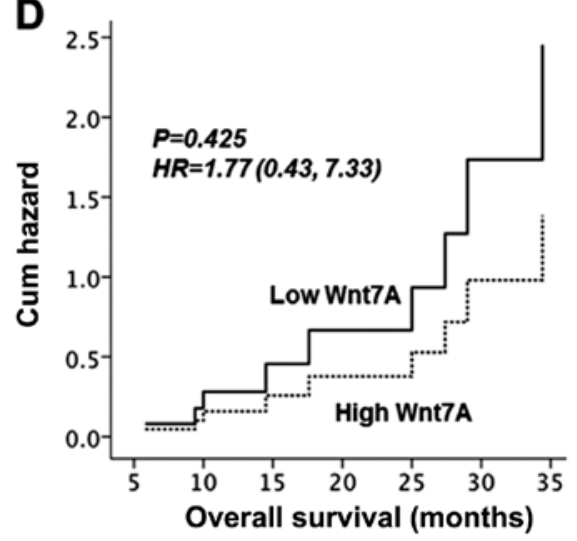

F

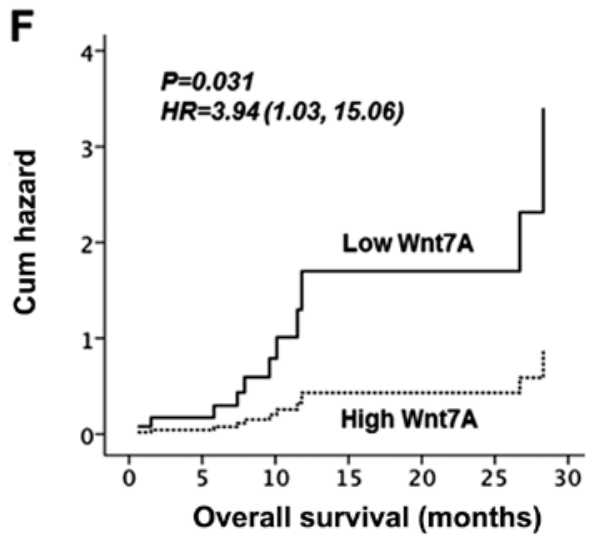

Figure 4. Hazard curves stratified by neoadjuvant chemotherapy and Wnt7A expression. Overall survival according to (A) neoadjuvant chemotherapy, (B) Wnt7A expression. (C) Overall survival of patients with high Wnt7A tumors according to neoadjuvant chemotherapy. (D) Overall survival of patients with neoadjuvant chemotherapy according to Wnt7A expression. (E) Overall survival of patients with low Wnt7A tumors according to neoadjuvant chemotherapy. (F) Overall survival of patients without neoadjuvant chemotherapy according to Wnt7A expression.

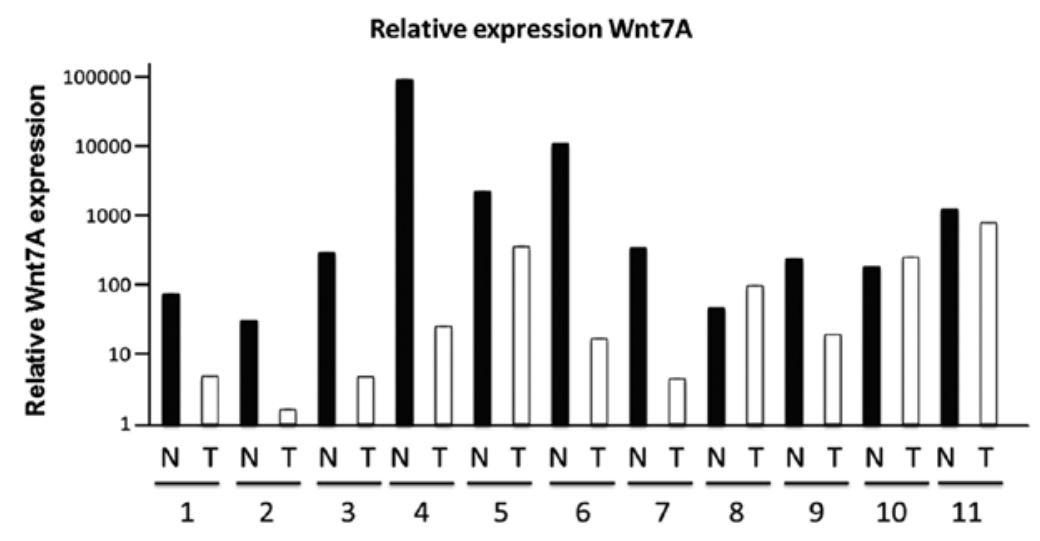

Figure 5. Wht7A expression differed significantly between the 11 paired normal (N) and tumor tissues (T). 
A

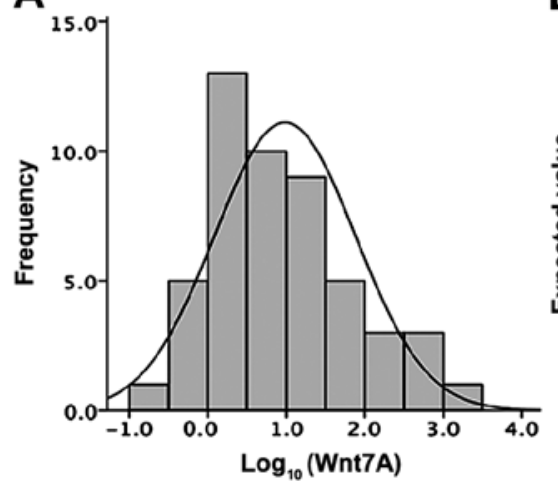

B

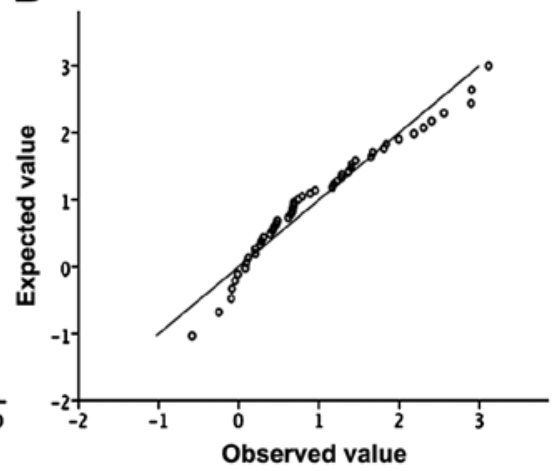

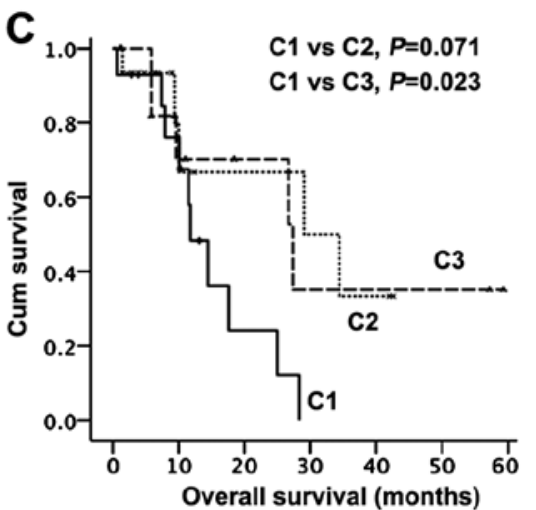

Figure 6. Statistical analysis of Wnt7A expression. (A) Frequency distribution of Wnt7A expression value logarithm. (B) Normal probability plot showing conformity of a normal distribution. (C) The Kaplan-Meier curves plotted for epithelioid tumors showed better prognosis $(\mathrm{C} 1$, low Wnt7A; 2 , intermediate Wnt7A; $\mathrm{C}$, high Wnt7A; $\mathrm{C} 1$ vs $\mathrm{C} 2, \mathrm{P}=0.071 ; \mathrm{C} 1$ vs $\mathrm{C} 3, \mathrm{P}=0.023)$.

is known to guide the development of the anterior-posterior axis in the female reproductive tract, and to play a critical role in uterine smooth muscle patterning and maintenance of adult uterine function. This gene is also responsive to changes in the levels of sex steroid hormone in the female reproductive tract. An inverse association for mRNA expression was found between Wnt7A and estrogen receptor $\alpha$ (ER- $\alpha)$ (34). Hypersensitivity of leiomyoma cells to estrogen may deregulate Wnt7A expression (34). Decreased Wnt7A expression may lead to loss of control in the patterning of the myometrium and result in the development of leiomyoma (34). In particular, the recent finding that ER- $\beta$ acts as a tumor suppressor has great potential relevance to predicting disease progression and therapeutic response in patients with MPM (35).

E-cadherin induction by $\mathrm{Wnt} / \beta$-catenin signaling is an evolutionarily conserved pathway operative in lung cancer cells, and loss of Wnt7A expression may be important in lung cancer development or progression through its effects on E-cadherin. Apparent physiologic levels of Wnt7A positively regulate E-cadherin expression in lung cancer (24).

During development, the Wnt pathway affects cell fate, polarity and proliferation, and Wnt7A has been implicated in the maintenance of HOX expression. In contrast to what occurs in normal lung and mortal short-term bronchial epithelial cultures, Wnt7A was frequently less expressed or absent in lung cancers. It is possible that those genes that are normally expressed in undifferentiated cells are upregulated in cancer, similar with other Homeobox genes $(23,36)$.

In our study, Wnt7A expression was highly correlated with IMIG stage (Fig. 1A). The primary tumor (T) seemed to contribute to stage than lymph node metastasis (N) (data not shown). These results indicate that the role of Wnt7A is to have cells differentiated and kept normal or restored to original specific morphology of the organ, and if it is lost or decreased, it may have consequence for worse prognosis in MPM.

In our study, Wnt7A expression might have been affected by smoking in normal or precancerous events such K-ras mutation, loss of EGFR mutation, or P53 mutation, even in pleural tissues. We found that MPM patients with a smoking history had lower Wnt7A expression than those who did not (Fig. 1C). Smoking considerably increases the risk of developing mesothelioma. A smoker who is exposed to asbestos has a 50- to 90 -fold greater chance of developing mesothelioma whereas a non-smoker exposed to asbestos has a 5 -fold greater chance $(37,38)$. It is possible that smoking might decrease Wnt7A expression associated with tumor-associated macrophages (39).

The results of our univariate analysis showed that gender, Eastern Cooperative Oncology Group performance status (ECOG PS), surgical procedure, and Wnt7A expression were not significant predictors of overall survival. With the exception of performance status, these findings are identical to those reported previously (7-10). ECOG PS, usually one of the most reliable prognostic factors, was not a significant predictor of survival in our study. However, ECOG PS data were not available for more than half of our patients, so we could not compare the relative contribution of ECOG PS to prognosis with that of Wnt7A expression. We did find that Wnt7A expression and histological subtype were similarly prognostic in the Cox's model, indicating that they could be prognostic markers for MPM.

The use of neoadjuvant chemotherapy before surgery for MPM has recently become common. However, neoadjuvant chemotherapy remains investigational because it has not definitively showed a survival benefit (40-42). A recent multicenter phase II trial using pemetrexed plus cisplatin neoadjuvant chemotherapy followed by extrapleural pneumonectomy (EPP) and hemithoracic radiation demonstrated feasibility and a reasonable long-term survival rate (6). On the other hand, neoadjuvant chemotherapy appeared to affect compliance with surgery and radiation therapy. These findings point to the need to determine which patients will benefit from neoadjuvant chemotherapy before surgery. The European Organization for Research and Treatment of Cancer (EORTC) prognostic score (EPS) for MPM, based on three consecutive phase II trials using different chemotherapy agents, found no association between objective tumor response and EPS classification (30). In our study, the survival analysis of 42 patients with epithelioid tumors showed that overall survival for the 20 patients who received neoadjuvant chemotherapy was not significantly better than that for 22 patients who did not. In the patients with high Wnt7A epithelioid tumors, overall survival did not differ between those who underwent neoadjuvant chemotherapy and those who did not $(\mathrm{P}=0.902$, Fig. 4C) while 
in patients with low Wnt7A epithelioid tumors, overall survival was significantly better in those who underwent neoadjuvant chemotherapy than in those who did not. These results indicate the patients with low Wnt7A expression do benefit, while patients with high $W n t 7 A$ expression do not benefit. Thus, we recommend neoadjuvant chemotherapy for patients with low Wnt7A tumors.

Among patients who did not have neoadjuvant chemotherapy, those with high Wnt7A expression had a significantly better prognosis than those with low Wnt7A expression. However, among patients who did have neoadjuvant chemotherapy, those with low Wnt7A expression had a significantly better prognosis than those with high $W n t 7 A$ expression. This indicates that $W n t 7 A$ is a putative novel prognostic factor for MPM and a novel predictor for determining whether neoadjuvant chemotherapy will be beneficial for patients with MPM. This means that patients with high Wnt7A expression have better prognosis, and patients with high $W n t 7 A$ expression might be more sensitive to neoadjuvant chemotherapy and they could have a better prognosis than those without neoadjuvant chemotherapy.

In conclusion, our study revealed that $W n t 7 A$ is a putative novel prognostic factor for MPM and a novel predictor for determining whether neoadjuvant chemotherapy will be beneficial for patients with MPM. Finally, our results suggest that $W n t 7 A$ is possibly a novel tumor-suppressor gene in MPM.

\section{Acknowledgements}

The present study was supported by NIH/NCI R01CA125030, and the Eileen D. Ludwig Endowed Fund for Thoracic Oncology Research (to B.H.); the Kazan, McClain, Abrams, Fernandez, Lyons, Greenwood, Harley and Oberman Foundation; the Jeffrey and Karen Peterson Family Foundation; and Paul and Michelle Zygielbaum Research Fund (to D.J.).

\section{References}

1. Robinson BW and Lake RA: Advances in malignant mesothelioma. N Engl J Med 353: 1591-1603, 2005.

2. Musk AW and de Klerk NH: Epidemiology of malignant mesothelioma in Australia. Lung Cancer 45: S21-S23, 2004.

3. Vogelzang NJ, Rusthoven JJ, Symanowski J, et al: Phase III study of pemetrexed in combination with cisplatin versus cisplatin alone in patients with malignant pleural mesothelioma. J Clin Oncol 21: 2636-2644, 2003.

4. Borasio P, Berruti A, Bille A, et al: Malignant pleural mesothelioma: clinicopathologic and survival characteristics in a consecutive series of 394 patients. Eur J Cardiothorac Surg 33: 307-313, 2008.

5. Flores RM, Zakowski M, Venkatraman E, et al: Prognostic factors in the treatment of malignant pleural mesothelioma at a large tertiary referral center. J Thorac Oncol 2: 957-965, 2007.

6. Krug LM, Pass HI, Rusch VW, et al: Multicenter phase II trial of neoadjuvant pemetrexed plus cisplatin followed by extrapleura pneumonectomy and radiation for malignant pleural mesothelioma. J Clin Oncol 27: 3007-3013, 2009.

7. Herndon JE, Green MR, Chahinian AP, Corson JM, Suzuki Y and Vogelzang NJ: Factors predictive of survival among 337 patients with mesothelioma treated between 1984 and 1994 by the Cancer and Leukemia Group B. Chest 113: 723-731, 1998.

8. Neumann V, Rutten A, Scharmach M, Muller KM and Fischer M: Factors influencing long-term survival in mesothelioma patientsresults of the German mesothelioma register. Int Arch Occup Environ Health 77: 191-199, 2004.

9. Metintas M, Metintas S, Ucgun I, et al: Prognostic factors in diffuse malignant pleural mesothelioma: effects of pretreatment clinical and laboratory characteristics. Respir Med 95: 829-835, 2001.
10. Baas P: Predictive and prognostic factors in malignant pleural mesothelioma. Curr Opin Oncol 15: 127-130, 2003.

11. Kumar-Singh S, Jacobs W, Dhaene K, et al: Syndecan-1 expression in malignant mesothelioma: correlation with cell differentiation, WT1 expression, and clinical outcome. J Pathol 186: 300-305, 1998.

12. Edwards JG, Swinson DE, Jones JL, Waller DA and O'Byrne KJ: EGFR expression: associations with outcome and clinicopathological variables in malignant pleural mesothelioma. Lung Cancer 54: 399-407, 2006.

13. Edwards JG, Faux SP, Plummer SM, et al: Cyclooxygenase-2 expression is a novel prognostic factor in malignant mesothelioma. Clin Cancer Res 8: 1857-1862, 2002.

14. Lopez-Rios F, Chuai S, Flores R, et al: Global gene expression profiling of pleural mesotheliomas: overexpression of aurora kinases and $\mathrm{P} 16 / \mathrm{CDKN} 2 \mathrm{~A}$ deletion as prognostic factors and critical evaluation of microarray-based prognostic prediction. Cancer Res 66: 2970-2979, 2006.

15. Gordon GJ, Rockwell GN, Godfrey PA, et al: Validation of genomics-based prognostic tests in malignant pleural mesothelioma. Clin Cancer Res 11: 4406-4414, 2005.

16. Shimizu E, Coxon A, Otterson GA, et al: RB protein status and clinical correlation from 171 cell lines representing lung cancer, extrapulmonary small cell carcinoma, and mesothelioma. Oncogene 9: 2441-2448, 1994.

17. Modi S, Kubo A, Oie H, Coxon AB, Rehmatulla A and Kaye FJ: Protein expression of the RB-related gene family and SV40 large $\mathrm{T}$ antigen in mesothelioma and lung cancer. Oncogene 19: 4632-4639, 2000.

18. Giacinti C and Giordano A: RB and cell cycle progression. Oncogene 25: 5220-5227, 2006.

19. Cote RJ, Jhanwar SC, Novick S and Pellicer A: Genetic alterations of the p53 gene are a feature of malignant mesotheliomas. Cancer Res 51: 5410-5416, 1991.

20. Wong L, Zhou J, Anderson D and Kratzke RA: Inactivation of p16INK4a expression in malignant mesothelioma by methylation. Lung Cancer 38: 131-136, 2002.

21. Yang CT, You L, Yeh CC, et al: Adenovirus-mediated p14(ARF) gene transfer in human mesothelioma cells. J Natl Cancer Inst 92: 636-641, 2000.

22. Logan CY and Nusse R: The Wnt signaling pathway in development and disease. Annu Rev Cell Dev Biol 20: 781-810, 2004.

23. Boersma CJ, Bloemen M, Hendriks JM, van Berkel EA, Olijve W and van Zoelen EJ: Homeobox proteins as signal transduction intermediates in regulation of NCAM expression by recombinant human bone morphogenetic protein-2 in osteoblast-like cells. Mol Cell Biol Res Commun 1: 117-124, 1999.

24. Ohira T, Gemmill RM, Ferguson K, et al: WNT7a induces E-cadherin in lung cancer cells. Proc Natl Acad Sci USA 100: 10429-10434, 2003.

25. Riddle RD, Ensini M, Nelson C, Tsuchida T, Jessell TM and Tabin C: Induction of the LIM homeobox gene Lmx1 by WNT7a establishes dorsoventral pattern in the vertebrate limb. Cell 83: 631-640, 1995

26. Timmreck LS, Pan HA, Reindollar RH and Gray MR: WNT7A mutations in patients with Mullerian duct abnormalities. J Pediatr Adolesc Gynecol 16: 217-221, 2003.

27. Bui TD, Lako M, Lejeune S, et al: Isolation of a full-length human WNT7A gene implicated in limb development and cell transformation, and mapping to chromosome 3p25. Gene 189: 25-29, 1997.

28. Kirikoshi $\mathrm{H}$ and Katoh M: Expression of WNT7A in human normal tissues and cancer, and regulation of WNT7A and WNT7B in human cancer. Int J Oncol 21: 895-900, 2002.

29. Raz DJ, Ray MR, Kim JY, et al: A multigene assay is prognostic of survival in patients with early-stage lung adenocarcinoma. Clin Cancer Res 14: 5565-5570, 2008.

30. Fennell DA, Parmar A, Shamash J, et al: Statistical validation of the EORTC prognostic model for malignant pleural mesothelioma based on three consecutive phase II trials. J Clin Oncol 23: 184-189, 2005.

31. Miller C, Degenhardt K and Sassoon DA: Fetal exposure to DES results in de-regulation of Wnt7a during uterine morphogenesis. Nat Genet 20: 228-230, 1998.

32. Mericskay M, Kitajewski J and Sassoon D: Wnt5a is required for proper epithelial-mesenchymal interactions in the uterus. Development 131: 2061-2072, 2004.

33. Parr BA and McMahon AP: Sexually dimorphic development of the mammalian reproductive tract requires Wnt-7a. Nature 395: 707-710, 1998. 
34. Li S, Chiang TC, Davis GR, Williams RM, Wilson VP and McLachlan JA: Decreased expression of Wnt7a mRNA is inversely associated with the expression of estrogen receptoralpha in human uterine leiomyoma. J Clin Endocrinol Metab 86: 454-457, 2001

35. Pinton G, Brunelli E, Murer B, et al: Estrogen receptor-beta affects the prognosis of human malignant mesothelioma. Cancer Res 69: 4598-4604, 2009.

36. Abate-Shen C: Deregulated homeobox gene expression in cancer: cause or consequence? Nat Rev Cancer 2: 777-785, 2002.

37. Andujar P, Wang J, Descatha A, et al: p16INK4A inactivation mechanisms in non-small-cell lung cancer patients occupationally exposed to asbestos. Lung Cancer 67: 23-30, 2010.

38. Becklake MR, Thomas D, Liddell F and McDonald JC: Follow-up respiratory measurements in Quebec chrysotile asbestos miners and millers. Scand J Work Environ Health 8: 105-110, 1982.
39. Coussens LM and Werb Z: Inflammation and cancer. Nature 420: 860-867, 2002.

40. Flores EM: Induction chemotherapy, extrapleural pneumonectomy, and radiotherapy in the treatment of malignant pleural mesothelioma: the Memorial Sloan-Kettering experience. Lung Cancer 49: S71-S74, 2005.

41. Tsao AS, Mehran R and Roth JA: Neoadjuvant and intrapleural therapies for malignant pleural mesothelioma. Clin Lung Cancer 10: 36-41, 2009.

42. Weder W, Kestenholz P, Taverna C, et al: Neoadjuvant chemotherapy followed by extrapleural pneumonectomy in malignant pleural mesothelioma. J Clin Oncol 22: 3451-3457, 2004. 